\title{
La implantación del sistema nacional de educación en Palencia. La aportación de los Ayuntamientos liberales
}

\section{The Implementation of the National Education System in Palencia. The Contribution of the Liberal City Councils}

\author{
FCO. JAVIER DE LA CRUZ MACHO \\ DIRECTOR IES VIRGEN DE LA CALLE (PALENCIA) \\ CTRA. SANTANDER, 1A, 34003 PALENCIA \\ fjcruzma@gmail.com \\ Recibido/Aceptado: 12/09/2020 \\ Cómo citar: De la Cruz Macho, J. (2021). La implantación del sistema nacional de educación en Palencia. \\ La aportación de los Ayuntamientos liberales. Tabanque: Revista pedagógica, 33, 17-29. \\ DOI: https://doi.org/10.24197/trp.1.2021.17-29
}

Resumen: La construcción del Sistema Nacional de Educación recayó, en el plano económico y de infraestructuras, en la gestión municipal. El Ayuntamiento palentino, a pesar de las dificultades económicas y los vaivenes políticos, afrontó este reto como una prioridad, dotando a la ciudad de infraestructuras, personal y recursos entre 1808 y 1936. Aunque el momento de mayor auge fue el último cuarto del siglo XIX, no por ello se escatimaron esfuerzos a lo largo de todo el periodo para convertir a Palencia en una ciudad con unos niveles de alfabetización superiores a la media y con unas instalaciones educativas, en parte, de elevada calidad constructiva y pedagógica.

Palabras clave: Ayuntamiento, Inversión, Liberalismo, Sistema Nacional de Educación, Escuelas.

\begin{abstract}
The construction of the National Education System fell, on the economic and infrastructure level, on municipal management. The City Council of Palencia, despite economic difficulties and political ups and downs, faced this challenge as a priority, providing the city with infrastructure, personnel and resources between 1808 and 1936. Although the moment of greatest boom was the last quarter of the nineteenth century, no efforts were spared throughout the period to turn Palencia into a city with higher than average literacy levels, and with educational facilities that were, in part, of high constructive and pedagogical quality.
\end{abstract}

Keywords: City government, Investment, Liberalism, National Educational System, Schools.

Sumario: Introducción; El origen de las primeras escuelas públicas. Ilustración y Liberalismo; El reinado de Isabel II. Periodo de consolidación y ampliación; El Sexenio Revolucionario. Nuevas iniciativas en un periodo convulso; De 1875 a 1902. Más y mejores escuelas; Cerrando el círculo educativo. 1902-1931; Conclusiones; Bibliografía.

Summary: Introduction; The origin of the first public schools. Enlightenment and Liberalism; The reign of Isabel II. Consolidation and expansion period; The Revolutionary Sexennial. New initiatives in a troubled period; From 1875 to 1902 . More and better schools; Closing the educational circle. 19021931; Conclusions; Bibliography. 


\section{INTRODUCCIÓN}

El inicio del liberalismo supuso, también, la implantación de un sistema nacional de Educación. Nacía, así, un sistema educativo que se iba a caracterizar por su progresiva secularización, en tanto que transfería parte de las actividades educativas de la Iglesia al Estado, por la libertad de enseñanza, que no podemos entender de forma plena, sino procesual, y por su sistematización, al ir pasando del "mosaico" educativo, a un sistema nacional unificado y jerarquizado (Tiana Ferrer, Ossenbahc Sauter y Sanz Fernández, 2002).

Este proceso va a quedar en manos, en lo referente a la enseñanza elemental o primaria, de los Ayuntamientos. ${ }^{1}$ En ellos recayó la responsabilidad de creación, impulso, desarrollo y mantenimiento del sistema escolar nacional.

Cabe, entonces, preguntarse por el papel que jugó el Ayuntamiento palentino en ese esfuerzo y en qué medida hizo, de esta preocupación liberal, una prioridad o, por el contrario, fue tan sólo una tarea más entre las múltiples que tuvieron que asumir los gobiernos municipales en el complejo y difícil proceso de construcción del Estado Liberal.

\section{EL ORIGEN DE LAS PRIMERAS ESCUELAS PÚBLICAS. ILUSTRACIÓN Y LIBERALISMO}

Conocer la evolución de la educación en Palencia, anterior al siglo XIX, no es cosa sencilla. La información es escasa y dispersa (Rodríguez Salcedo, 1949). Lo que sí sabemos con certeza es que, desde los inicios de la Edad Media, se caracterizaba por su vinculación a la Iglesia y su carácter asistencial (Labrador Herráiz y Nieto Bedoya, 1990).

A partir de los estudios de que disponemos sabemos que, hasta el siglo XIX, perviven en Palencia el Seminario Conciliar, y el Colegio de los Niños de la Doctrina Cristiana. El primero vinculado a la formación de sacerdotes y el segundo de carácter asistencial (González Gallego, 1987 y 1990). Desde su creación, el Ayuntamiento mantuvo un patronato sobre el colegio de los Doctrinos, destinando unas cantidades anuales a su mantenimiento (González Gallego, 1990).

El catastro de Ensenada refiere la existencia en Palencia, en 1749, de 7 maestros (Labrador Herráiz y Nieto Bedoya, 1987) y un preceptor de gramática (Nieto Bedoya, 1993), de los que, una parte, eran sostenidos directamente por el Ayuntamiento. Lo que no suponía la existencia de una escuela municipal.

\footnotetext{
${ }^{1}$ El artículo 325, apartado quinto, de la Constitución de Cádiz situaba el "cuidar de todas las escuelas de primeras letras y de los demás establecimientos de educación que se paguen de los fondos del común" en el ámbito de las competencias y responsabilidades municipales. No varió esta situación ni con la ley de instrucción primaria de 1838, ni con la de Claudio Moyano de 1857, ni en las normativas posteriores.
} 
La implantación del sistema nacional de educación en Palencia. La aportación de los ayuntamientos liberales

De hecho, siguiendo a Gutiérrez Barba (1990), la primera escuela a cargo del Ayuntamiento palentino surgió en 1767, al hacerse cargo el municipio de la que regentaban los jesuitas tras su expulsión. Escuela que atendía los niveles más básicos y que, en 1789, se vio complementada con otra de carácter superior. La primera dedicada a leer y a la enseñanza de la doctrina cristiana y la segunda a escribir y contar.

Esta situación se mantiene en 1811, según informe recogido en el Ayuntamiento, en el que se especifica que: "Hay dos escuelas de primeras letras de la una es Patrona la Ciudad y se pagan a sus dos Maestros de propios y de la otra es patrona la Cofradía de Trinidad; hay también un Estudio de Rudimentos de Latinidad del que es Patrono el Cabildo". ${ }^{2}$ Un informe posterior ${ }^{3}$ especifica un poco más la situación al detallar la existencia de dos escuelas del Ayuntamiento, que antes pertenecían a los regulares de los Jesuitas. Una enseña a leer y los primeros rudimentos y la otra a escribir y contar, así como la doctrina cristiana. Además, hay otras dos escuelas, una que enseña a leer, escribir y contar por un maestro particular, al que pagan los padres de los alumnos y el colegio de los Niños de la Doctrina Cristiana. Además, existían dos maestros de latinidad que recibían un sueldo del Ayuntamiento, junto a aportaciones del alumnado (Álvarez García, 1987). Por último, el convento de San Francisco tenía también una escuela.

El hecho de que el Ayuntamiento se hiciese cargo de la escuela de los jesuitas, tras su expulsión, no significa que fuese la simple aceptación de una situación creada. Por el contrario, el gobierno municipal manifestó una honda preocupación por su escuela. De hecho, en 1770 el Ayuntamiento Palentino aprobó sus primeras ordenanzas sobre enseñanza primaria ${ }^{4}$ que perdurarán hasta la aprobación de unas nuevas en $1817 .{ }^{5}$ En estas últimas, entre otras cuestiones, se especificaba la edad de cuatro años y medio para empezar la escolarización y la gratuidad de la enseñanza.

El Ayuntamiento se preocupó, también, de garantizar el mantenimiento de los recursos económicos destinados a la enseñanza. En un contexto de crisis hacendística permanente, en el que los ingresos municipales eran escasos y las demandas de la hacienda estatal enormes, se produjeron varias incautaciones de impuestos municipales por parte del Estado. En la acaecida en 1832, el Ayuntamiento solicitó que no se incautasen los impuestos en su totalidad, ya que de ellos dependía el pago "del mantenimiento de las Escuelas y sueldos de

\footnotetext{
${ }^{2}$ Archivo Municipal de Palencia. (en adelante AMP). 17-9-1811.

${ }^{3}$ AMP, Actas Municipales. 7-5-1816.

${ }^{4}$ AMP, Actas Municipales. 26-8-1817.

${ }^{5}$ AMP, Actas Municipales, 26-8-1817.
} 
maestros". ${ }^{6}$ Simultáneamente el Ayuntamiento procuraba dotar, dentro de sus posibilidades, del mobiliario necesario a sus escuelas. ${ }^{7}$

Fue en 1816 cuando apareció, en Palencia, la primera escuela de niñas, fundada por la Sociedad Económica de Amigos del País de Palencia, que se hizo cargo de la mayor parte de los gastos (Gutiérrez Barba, 1990). Sin embargo, en marzo de 1822, la Económica comunicó al Ayuntamiento que no podía seguir manteniendo la escuela de niñas, ni la de dibujo, solicitando al Ayuntamiento se hiciese cargo de ellas. El Ayuntamiento se responsabilizará de su sostenimiento ${ }^{8}$, dotando a la escuela con 5.000 reales, para pagar el sueldo de la maestra, Francisca Casasola, un maestro de primeras letras y una ayudanta (Gutiérrez Barba, 1990). En ese momento asistían a la escuela 90 niñas.

Palencia termina este periodo con tres escuelas municipales, dos de niños y una de niñas. Al igual que en otras ciudades españolas (Delgado Criado 1994), los últimos ayuntamientos absolutistas, y los primeros liberales, mejoraron la situación educativa.

\section{EL REINADO DE ISABEL II. PERIODO DE CONSOLIDACIÓN Y AMPLIACIÓN}

El inicio del reinado de Isabel II fue convulso. Por eso los primeros años fueron de una cierta atonía, también a nivel municipal. Sin embargo, a mediados de los 40 se vivió un periodo dorado para la educación con tres grandes actuaciones. Por un lado, se creó una nueva escuela municipal bajo la alcaldía de Eduardo Rodríguez Cossío, en el arrabal de Paredes de Montes. ${ }^{9}$ La creación de esta escuela tiene un importante significado, ya que no sólo supone dotarse de una cuarta escuela sostenida por el Ayuntamiento, sino que ésta se crea en una de las zonas más pobres de la ciudad, extramuros a la misma, una pequeña localidad dentro del término municipal. Constituye un esfuerzo por llevar la educación a todos los sectores de la población.

La segunda actuación tiene que ver con la enseñanza secundaria. En 1843, bajo la alcaldía de Mariano Garrido, se creó una comisión, impulsada desde el Ayuntamiento, para poner en marcha un instituto de Secundaria en la ciudad. ${ }^{10}$ Su objetivo: buscar profesorado, material y edificio. ${ }^{11}$ Poco más tarde el Instituto será una realidad, contando en esa ocasión con el apoyo de la legislación estatal. La creación del Instituto supuso la incorporación al mismo de las dos escuelas de latinidad existentes en la ciudad mantenidas por el Ayuntamiento ${ }^{12}$ (Álvarez

\footnotetext{
${ }^{6}$ AMP, Actas Municipales, 16-5-1832.

${ }^{7}$ AMP, Actas Municipales, 7-5-1816.

${ }^{8}$ AMP, Actas Municipales, 8-3-1822.

${ }^{9}$ AMP, Actas Municipales, 22-6 y 14-8 de 1844.

${ }^{10}$ AMP, Actas Municipales, 1-4-1843.

${ }^{11}$ AMP, Actas Municipales, 2-8-1843.

${ }^{12}$ AMP, Actas Municipales, 22-1-1845.
} 
La implantación del sistema nacional de educación en Palencia. La aportación de los ayuntamientos liberales

García, 1997). El lugar donde se ubicó fue el desamortizado convento de San Buenaventura. ${ }^{13}$

El Ayuntamiento se hizo cargo de la adecuación del edificio y de su posterior mantenimiento, así como de las continuas reformas que hubo que acometer en el mismo. Además, colaboró en la financiación de este aportando anualmente una cantidad (Álvarez García, 1987) y con la concesión de becas para alumnos.

También venía prestando atención el Ayuntamiento, a la educación de párvulos. ${ }^{14}$ El primer paso lo dio la iniciativa privada al crearse, en 1857, una escuela de párvulos. ${ }^{15}$ El Ayuntamiento se implicó desde el inicio en la misma, dotando de becas a alumnos para que fuesen acogidos en dicha escuela. Cuatro años después de su fundación, la escuela pasó a ser de titularidad municipal, ${ }^{16}$ asumiendo el Ayuntamiento el coste íntegro de su mantenimiento, especialmente el del sueldo del maestro y la dotación de materiales. ${ }^{17}$

La asunción por parte del Ayuntamiento de la Escuela de Párvulos supuso el cambio de ubicación, abandonando el edificio de la calle Ramírez, para instalarse en el antiguo edificio de los Niños de la Doctrina Cristiana que el Ayuntamiento había comprado tras ser suprimida dicha escuela en 1850 (Espinilla Herrarte y González Sánchez, 2007).

La tercera actuación tiene que ver con los estudios de magisterio. En 1841 el gobierno había publicado una orden para establecer una Escuela Normal de Prácticas de Primaria en cada provincia. ${ }^{18}$ El Ayuntamiento inició el proceso de forma inmediata. El primer paso fue solicitar su creación, lo que se retrasará, ya que la concesión de esta no se recibirá hasta el 9 de septiembre de $1846 .{ }^{19}$ Unos

\footnotetext{
13 "E1 único lugar disponible en Palencia era el ex-convento de San Buenaventura, de la orden de los Descalzos, expropiado a raíz de los Decretos de Mendizábal (1835 y 36) y, por ello, perteneciente a la Nación. Estaba situado en la zona oeste de Palencia, a orillas del río Carrión. Aunque no reunía las condiciones necesarias para ser dedicado a Instituto, dada la altura de sus techos, sí era muy apropiado para el internado: Edificio independiente, con plazuela, huerto, cercado y 'su famoso argive'. De todas formas, se acondicionaron cinco aulas, y pudo comenzarse el curso el 1 de diciembre de 1845." (Álvarez García, 1987, p. 348)

${ }^{14}$ De hecho, en las actas municipales, aparece varias veces esta demanda. AMP, Actas Municipales. 283-1846; 16-11-1850, 22-12-1850,

${ }^{15}$ La escuela, iniciada en 1857, no recibió la autorización oficial hasta febrero de 1858. AMP, Actas Municipales 24-2-1858. Era una escuela mixta, de niños y niñas.

16 "La implicación del Ayuntamiento en el sostenimiento y evolución de esta escuela fue evidente e importante para mantenerse a lo largo del tiempo. El propio alcalde D. Pablo Espinosa concedió 2.000 reales para que se admitieran a 20 niños pobres, enviados desde el Ayuntamiento. Por otra parte, en 1860, tres años después de abrir sus puertas, pasará a ser considerada como la primera Escuela Municipal de Párvulos de Palencia. La nueva situación institucional de la escuela, ahora municipal, supuso cambios en su ubicación, pasando de la calle Ramírez al edificio de la calle de los Doctrinos, y otorgando a los maestros un sueldo de 5.000 reales". (Nieto Bedoya 2006, p. 845)

${ }^{17}$ AMP, Actas Municipales, 9-4-1862. Esta actuación municipal derivaba de una orden ministerial de 1853 que ordenaba la creación de asilos de párvulos en cada capital de provincia.

${ }^{18}$ AMP, Actas Municipales, 16-2-1841.

${ }^{19}$ AMP, Actas Municipales, 9-9-1846.
} 
días más tarde, el 30 de septiembre, se comunicaba la aprobación del presupuesto para su creación..$^{20}$ Sin embargo, la escuela no será una realidad hasta febrero de 1861 (García Colmenares, 1987). Instalada, inicialmente, en el Instituto de Secundaria, se trasladará, posteriormente, al edificio de la Tarasca. ${ }^{21}$ Ello conllevaba la creación de la correspondiente escuela práctica o aneja a cargo del Ayuntamiento.

Además de estos proyectos Palencia vivió también el dulce sueño de un nuevo centro educativo. De la mano del ministro de Fomento Agustín Esteban Collantes, Palencia recibió en 1854 la noticia de la creación de un colegio de sordomudos en la ciudad. ${ }^{22}$ El Ayuntamiento se puso en marcha con gran celeridad, para ofrecer un local. ${ }^{23}$ Sin embargo, la llegada del Bienio Progresista, y el exilio a Francia del ministro palentino, impidieron su realización. Finiquitado el Bienio la orden de creación del colegio de sordomudos se revocó.

A todo este esfuerzo se sumarán diferentes reformas y arreglos para mejorar los locales, trasladando la escuela de niñas y la de párvulos al cuartel de la Tarasca. A la vez, la escuela de niñas, sostenida por el Ayuntamiento, seguía estando bajo titularidad de la Sociedad Económica, algo a lo que se pondrá fin en este periodo, pasando a ser de titularidad municipal, ${ }^{24}$ cambio meramente nominal, pues su pleno sostenimiento corría, hacía tiempo, a cargo de los fondos municipales.

Vemos, por lo tanto, cómo en la etapa isabelina se extiende y consolida el sistema escolar nacional, convirtiéndose la educación en una de las principales preocupaciones del gobierno municipal, que no escatimará recursos ni esfuerzos para su mantenimiento y desarrollo, en sintonía con la evolución del resto de ciudades de Castilla y León (Hernández Díaz, 1993).

\section{EL SEXENIO REVOLUCIONARIO. NUEVAS INICIATIVAS EN UN PERIODO CONVULSO}

Durante este periodo, dos son las medidas que toma el Ayuntamiento para el impulso educativo: la creación de una Escuela Nocturna de Adultos y una Biblioteca Popular, en un intento de ofertar espacios formativos para la población adulta obrera. Suponía también, un esfuerzo por ofrecer una alternativa a la oferta eclesial, que mantenía una escuela de adultos impulsada por la Propaganda

\footnotetext{
${ }^{20}$ AMP, Actas Municipales, 30-9-1846.

${ }^{21}$ AMP, Actas Municipales, 1-4-1863. Se da cuenta del inicio de las obras de reforma del edificio de la Tarasca para albergar la Escuela Normal.

${ }^{22}$ AMP, Actas Municipales, 14 y 16-1-1854.

${ }^{23}$ Primero en la calle Don Sancho, propiedad del duque de Gor, cuyas condiciones exigidas por su dueño no convencieron al consistorio (AMP, Actas Municipales, 29-3-1854.) Después se optó por arreglar el local de las escuelas del primer distrito en la calle Ramírez, cuyas obras estaban culminadas a finales de 1854, AMP, Actas Municipales, 8-4-1854.

${ }^{24}$ AMP, Actas Municipales, 9-3-1850.
} 
La implantación del sistema nacional de educación en Palencia. La aportación de los ayuntamientos liberales

Católica. Este hecho se enmarca en un proceso de secularización más amplio impulsado desde el Ayuntamiento (Cruz Macho, 2019). Debajo de este proyecto latía también el convencimiento de la importancia de la educación para el desarrollo de la sociedad. ${ }^{25}$ Ya existía un embrión de escuela de Adultos, pues clases para este colectivo se impartían en la Normal ${ }^{26}$ asumiendo el coste el gobierno municipal. Desde 1869 el Ayuntamiento asumirá esta tarea como propia, haciéndose cargo de manera oficial. No se conformó con crear la escuela, sino que trató de impulsar la asistencia del alumnado a estos centros, dictaminando que para los puestos municipales se preferiría a los que tuviesen un informe de sus profesores de la Escuela de Adultos, ${ }^{27}$ a la vez que se establecieron una serie de premios para el alumnado. ${ }^{28}$ Posteriormente la escuela de adultos se desligará totalmente de la Normal, iniciando su andadura de forma independiente, en noviembre de $1870 .{ }^{29}$ La iniciativa dio resultados ya que en 1870 se matricularon doscientas personas. Se iniciaba así un camino que trataba de garantizar la educación básica a toda la sociedad. ${ }^{30}$ Esta escuela estaría activa hasta 1873 , cuando se cierra, volviéndose a abrir en 1875 , hasta que, en 1883 , se una a la de la Propaganda Católica (Álvarez García, 1997).

Respecto a la instalación de la biblioteca popular el proyecto se inició a principios de 1870, a instancias del presidente de la junta provincial de primera enseñanza. La biblioteca se instaló en una de las escuelas existentes y se puso en marcha con libros que donó el Gobierno, aportando el Ayuntamiento el mobiliario.

\footnotetext{
25 "Una de las cosas con preferencia que deben llamar la atención del Municipio es, atender por todos los medios imaginarios á la instrucción y educación de sus semejantes, por lo cual se eleva el espíritu á las más bellas acciones de una sana moral, por este camino se conseguirá destruir la crasa ignorancia que con furor toma asiento en las clases necesitadas, haciéndolas concebir pensamientos mezquinos y hechos vituperables." AMP, Actas Municipales, 16-2-1870.

26 AMP, Actas Municipales, 30-12-1868. El director de la escuela Normal, Gonzalo Sanz, venía impartiendo estas clases gratuitas para el alumnado, corriendo con los gastos el Ayuntamiento.

27 "Que haga constar el Ayuntamiento que preferirá para los empleos que sean necesarios al municipio, á los que por su instrucción y buena conducta moral, vengan provistos de una credencial, diploma ó título, por el que conste el visto bueno de sus dignos profesores, de buen comportamiento, aplicación y buenas costumbres, en todas sus esferas morales; como así bien el Ayuntamiento al presenciar los exámenes anuales, interpondrá todo su valimiento para que sean recompensados los sobresalientes, teniéndolos en memoria para encomendarles en su arte ú oficio, cuantas obras fuesen de administración del Municipio, no rebajándoles en ningún concepto de los licenciados del ejercito...." Además, se aprueba la obligatoriedad de saber leer y escribir para poder acceder a un puesto municipal, aunque luego no se cumpla esa obligación. AMP, Actas Municipales, 16-2-1870.

${ }^{28}$ AMP, Actas Municipales, 14-5-1870.

${ }^{29}$ AMP, Actas Municipales, 9-11-1870.

30 "Antes de que acabe el siglo, este ideal casi se conseguirá en Palencia, llegando en 1895 a dedicar nada menos que el $12 \%$ de su presupuesto. En 1874 la ciudad palentina poseía cuatro escuelas elementales de niños; dos elementales de niñas; una de párvulos; y dos de beneficencia. Como escuelas privadas existían cuatro de niños y nueve o 10 establecimientos de niñas. Junto a éstas habría que añadir la de la Asociación de la Propaganda Católica, escuela de invierno, nocturna y dirigida a personas adultas, educándose en la ciudad algo más de 1.000 niños." (Nieto Bedoya, 2006, p. 819)
} 


\section{DE 1875 A 1902. MÁS Y MEJORES ESCUELAS}

En el inicio de la Restauración la educación constituyó una de las principales preocupaciones para el alcalde Juan Martínez Merino. Entre sus primeras propuestas, la creación de una escuela de párvulos de carácter municipal que atendiese a las/os hijas/os de los jornaleros y trabajadores, aunque concebida con un carácter más asistencial que educativo. ${ }^{31}$

Poco se tardaría en llevarlo a efecto ya que bastará con el alquiler de un local y la adquisición del mobiliario. En diciembre de 1876 ya se contaba con un profesor titular. Inicialmente se ubicó en la antigua escuela de los Doctrinos, en el entorno de San Miguel. A la vez se seguía manteniendo la otra escuela de párvulos de la que se convertirá en titular Vicente Inclán.

Antes que la escuela de párvulos, el alcalde había propuesto la puesta en marcha de una escuela de adultos, ${ }^{32}$ tras la desaparición de la anterior en 1873. Su proceso fue rápido, ya que se instaló en las dependencias de la Escuela Normal y el director de la misma se encargó de impartir las clases.

Bajo la alcaldía de Tadeo Ortiz se puso en marcha una escuela de Artes y Oficios, a propuesta del Ateneo, el 21 de enero de 1880. Su constitución corrió a cargo del Ateneo, limitándose el Ayuntamiento a apoyarla económicamente y a dotarla de premios para los trabajos de los mejores alumnos. ${ }^{33}$ Esta escuela tuvo una vida difícil debido a sus necesidades económicas, finalizando su andadura en 1886 continuando sólo la Escuela de Dibujo, bajo el paraguas municipal. A partir de ese momento, el Ayuntamiento colaboró con la Escuela de Artes y Oficios de la Propaganda Católica, mediante subvenciones o concesión de premios para los alumnos destacados. ${ }^{34}$

Pero el mayor esfuerzo municipal vino de la renovación de los edificios destinados a escuela. Muchos eran viejas casas alquiladas o antiguos locales reacondicionados, de pésimas condiciones y necesitados de continuos arreglos.

Ese era el caso de la antigua escuela de los Doctrinos, lo que motivó la construcción del primer grupo escolar, de planta rectangular con un patio interior porticado. En su diseño se cuidó que hubiese suficiente luz natural, y que los espacios fuesen amplios y holgados, además de disponer de habitaciones para los maestros, despachos y aseos, así como un pequeño jardín para los párvulos. El grupo escolar se inauguró oficialmente el 4 de septiembre de 1886 (Cruz Macho, 2016).

Años más tarde se propuso la creación de un nuevo grupo escolar en el barrio de La Puebla. En 1894 el Ayuntamiento compró las casas 21 de Corredera, 1 al

\footnotetext{
${ }^{31}$ AMP, Actas Municipales, 3-3-1875.

${ }^{32}$ AMP, Actas Municipales, 13-1-1875.

${ }^{33}$ AMP, Actas Municipales, 28-1-1880.

${ }^{34}$ AMP, Actas Municipales, 13-12-1889.
} 
La implantación del sistema nacional de educación en Palencia. La aportación de los ayuntamientos liberales

13 de la Plata y 2 al 4 de Rizarzuela. El 3 de septiembre de ese mismo año, 1894, en las fiestas de San Antolín, se colocó la primera piedra. En este caso el plano del edificio había sido diseñado por el arquitecto municipal Juan Agapito Revilla. Las obras se prolongaron durante tres años, concluyéndose a finales de 1897 (Calle Carracedo y Hernández del Campo, 1990).

También se construyó un nuevo edificio para escuelas en Paredes del Monte en 1879. ${ }^{35}$ La Escuela de Dibujo mejoró con la instalación de luz eléctrica y excusados.

\section{CERRANDO EL CÍRCULO EDUCATIVO. 1902-1931}

Tras el ingente esfuerzo, el siglo arrancaba con 13 escuelas municipales, repartidas en 4 distritos de niños, ${ }^{36} 2$ distritos de niñas, dos de párvulos, cuatro vinculadas a la enseñanza de Magisterio, y la de Paredes del Monte. (Espinilla Herrarte, 2010). A ella habría que añadir las dedicadas a la enseñanza de adultos. En 1904 se crearon tres secciones más, vinculadas a los estudios de Magisterio.

En este periodo la actuación más importante fue la creación del grupo escolar del tercer distrito, que vendría a unirse a los dos ya existentes El proceso para ese nuevo grupo escolar se inició en $1904,{ }^{37}$ aunque no se hizo realidad hasta 1930, ubicándose en el antiguo cuartel de Alfonso XII, reacondicionado para la nueva función. Ese mismo año se aprobó el nombre que iba a tener cada grupo escolar. ${ }^{38}$ El nuevo, se denominó Alonso Berruguete, y los otros dos recibieron los nombres de Modesto Lafuente y Jorge Manrique.

Pero siendo esta la obra más importante no fue la única que supuso una ampliación de la dotación en infraestructuras escolares, prestándose especial atención a los barrios periféricos de la ciudad. En 1904 se inició la reparación de las escuelas de Paredes del Monte, en esta ocasión con cargo a los presupuestos del Estado. En 1928 y tras tres años de proyectos ${ }^{39}$ y obras vieron la luz unas nuevas escuelas nacionales ubicadas en las huertas de Florentino Pombo, de donde tomaron su nombre, y en 1929 se alquiló un local en Santa Ana para ubicar en él las escuelas de Allende el Río. ${ }^{40}$

\footnotetext{
${ }^{35}$ AMP, Actas Municipales, 1-8-1879.

${ }^{36}$ El cuarto distrito surge al ser suprimida la auxiliar de la Normal de Maestros, surgiendo esa nueva escuela para continuar con la enseñanza de los niños en ella matriculados.

${ }^{37}$ AMP, Actas Municipales, 6-4 y 26-8 de 1904. En 1907 se recibió una subvención para la realización de dicho grupo escolar, (AMP, Actas Municipales, 6-9-1907), y en 1908 se prorrogó dicha subvención, (AMP, Actas Municipales, 10-7-1908) y nuevamente en 1909 (AMP, Actas Municipales, 22-1-1909), quedando anulada en 1910 (AMP, Actas Municipales, 5-8-1910). Habrá que esperar a 1919 a que se retome el proyecto (AMP, Actas Municipales, 12-3-1919).

${ }^{38}$ AMP, Actas Municipales. 3-5-1930.

${ }^{39}$ El Día de Palencia, 31-5-1926.

${ }^{40}$ AMP, Actas Municipales, 13-11-1929.
} 
La inversión en edificios no fue la única actuación, sino que las escuelas ya existentes vieron aumentadas sus secciones sobre todo durante los años finales de la Dictadura de Primo de Rivera. En 1930 Palencia contaba con 33 escuelas públicas y 19 privadas (Espinilla Herrarte, 2010).

De mayor transcendencia, al menos por la repercusión social, fue la construcción de un nuevo edificio para albergar el Instituto de Secundaria. Se abandonaba así el viejo convento de San Buenaventura y se construía un edificio moderno adaptado a las necesidades educativas. En este caso el Ayuntamiento cedió los terrenos. ${ }^{41}$

Se consiguió también realizar un viejo sueño decimonónico, la creación de una escuela de artes y oficios en la ciudad, sustituyendo a la antigua escuela de dibujo. Se puso en marcha en el convento de San Buenaventura en octubre de 1922, gracias a la cesión del Ayuntamiento del material y recursos de la Escuela de Dibujo. ${ }^{42}$ La disponibilidad de un nuevo edificio no será una realidad hasta 1946, cuando se traslade al antiguo dispensario antituberculoso.

\section{CONCLUSIONES}

La implantación y desarrollo de un sistema nacional de enseñanza, en su nivel elemental, quedó en manos de la iniciativa municipal. Los Ayuntamientos liberales palentinos asumieron ese reto y, tras 130 años de gobierno, extendieron la oferta educativa a toda la población urbana, junto a la enseñanza privada, asumiendo el gobierno municipal más de $2 / 3$ de la misma.

El balance global es positivo. Las élites políticas, que rigieron los destinos municipales, fijaron la educación como una de sus prioridades y a ella dirigieron multitud de esfuerzos e inversiones. Bien es cierto que el proceso pudo ser más rápido, que algunos proyectos se demoraron y que otros se iniciaron con retraso. Pero conviene no olvidar el contexto histórico de un periodo marcado por diferentes guerras, que mermaron los ingresos municipales en beneficio del Estado, además de los continuos vaivenes políticos Por otra parte, el Estado liberal y, por ende, los Ayuntamientos, carecieron de un sistema impositivo y recaudatorio suficiente para construir el nuevo modelo de Estado y dar respuesta a las nuevas demandas y servicios. De hecho, el Ayuntamiento palentino sufrió varias incautaciones de impuestos por parte del Estado, a la vez que tuvo que hacer frente a otros proyectos como la traída de aguas, el alumbrado eléctrico, la construcción de una plaza de Abastos, el ordenamiento urbano, el impulso al ferrocarril, o la construcción de su propia sede de gobierno municipal.

Teniendo en cuenta esas cuestiones, y a la luz del proceso reseñado en este artículo, es como podemos afirmar el importante papel del Ayuntamiento en el

\footnotetext{
${ }^{41}$ AMP, Actas Municipales, 29-7-1905.

${ }^{42}$ AMP, Actas Municipales, 25-11-1921 y 3-2-1922.
} 
La implantación del sistema nacional de educación en Palencia. La aportación de los ayuntamientos liberales

establecimiento del sistema nacional de enseñanza, su secularización y sistematización.

El Ayuntamiento fue ampliando, paulatinamente su oferta educativa, aumentando el número de escuelas. Además, la educación se extendió a todos los barrios, incluso a los periféricos, haciendo un esfuerzo por hacerla accesible a todas las clases sociales.

Por otra parte, en algunos casos (escuela de párvulos o de niñas), aunque las primeras iniciativas fueron ajenas al Ayuntamiento, los diferentes gobiernos municipales colaboraron en su puesta en marcha y sostenimiento y terminaron asumiéndolas, llegado el momento.

El Ayuntamiento colaboró también, en otras iniciativas educativas, ajenas a su campo de competencia. Prueba de ello fueron la escuela de dibujo, la inestable de adultos y de artes y oficios, la biblioteca popular, o u impulso a la enseñanza secundaria. Lo mismo podemos decir con el fallido proyecto de la escuela de sordos o con su apoyo a la Normal.

No podemos desconocer las dificultades, como la calidad de algunos locales, sobre todo hasta el inicio de la Restauración, o el no disponer de las escuelas necesarias en función de la población de la ciudad. Pero estos fueron problemas comunes en todo el territorio nacional fruto de un proceso que, prácticamente, partía de cero. Por otra parte, en cuanto la situación económica lo permitió el Ayuntamiento invirtió para solventar estos problemas. Buena muestra de ellos los dos grupos escolares, que recibieron elogios de las autoridades educativas.

En este sentido, que Palencia se situase como una de las ciudades con mayores tasas de alfabetización en España, tiene mucho que ver con la actuación de los gobiernos municipales, sin por ello negar otros factores y actuaciones.

\section{BIBLIOGRAFÍA}

Álvarez García, M. C. (1987). Origen, creación y funcionamiento del I.N.B. Jorge Manrique de Palencia. Publicaciones de la Institución Tello Téllez de Meneses, (57), 341-364.

Álvarez García, M. C. (1997). La Enseñanza Secundaria en Palencia durante el siglo XIX (1845-1901). Palencia: Publicaciones de la Institución Tello Téllez de Meneses.

Archivo Municipal de Palencia. Libro de Actas del Ayuntamiento. Años 18111930. 
Calle Carracedo, M. de la y Hernández Del Campo, R. I. (1990). La construcción de las escuelas públicas del barrio de la Puebla en el Paseo del Salón: Criterios didácticos y conceptos arquitectónicos escolares. Palencia 18941898. II Congreso de Historia de Palencia.

Cruz Macho, F. J. de la (4 de septiembre de 2016). 130 aniversario del colegio Jorge Manrique. Diario Palentino, pp.26-27.

Cruz Macho, F. J. de la (2019). Palencia, la ciudad y sus alcaldes. 1808-1936. Palencia: PITMM.

Delgado Criado, B. (coord.). (1994). Historia de la educación en España y América. La educación en la España Contemporánea (1789-1975). Madrid: SM.

El Día de Palencia. Prensa local.

Espinilla Herrarte, M. L. (2010). Escuelas, Alumnado y Docentes Palentinos en el Primer Tercio del Siglo XX. Valladolid: Autoedición.

Espinilla Herrarte, M. L. y González Sánchez, J. L. (2007). Génesis de la primera “escuela de párvulos" Vicente Inclán. Tabanque (20), 137-166.

García Colmenares, C. (1987). La Escuela Normal de Palencia. Apuntes Histórico-Pedagógicos (1861-1940). Ier. Congreso de Historia de Palencia.

González Gallego, I. (1987). El proyecto didáctico de una institución educativa entre los siglos XVI y XIX: El colegio de niños de la Doctrina Cristiana de Palencia (1544-1861). Ier. Congreso de Historia de Palencia.

González Gallego, I. (1990). Educación y Asistencia Social en Palencia: Un caso entre el siglo XVI y el XIX. II Congreso de Historia de Palencia.

Gutiérrez Barba, A. (1990). Una aproximación a la enseñanza pública palentina de las primeras letras, en el tránsito del siglo XVIII al XIX. II Congreso de Historia de Palencia.

Hernández Díaz, J. M. (1993). La escuela primaria en Castilla y León. Salamanca: Ediciones Amaru. 
La implantación del sistema nacional de educación en Palencia. La aportación de los ayuntamientos liberales

Labrador Herraiz, C. y Nieto Bedoya, M. (1987). La Escuela en el Antiguo Régimen. Los maestros de primeras letras en la provincia de Palencia. Ier. Congreso de Historia de Palencia.

Labrador Herráiz, C. y Nieto Bedoya, M. (1990). La Escuela en el Antiguo Régimen. Acciones Benéfico-Sociales y enseñanza en la provincia de Palencia. II congreso de Historia de Palencia.

Nieto Bedoya, M. (1991). La enseñanza de primeras letras en Castilla y León en el siglo XVIII: Palencia un ejemplo singular, en Hernández Díaz, J. M., La escuela primaria en Castilla y León, (pp. 9-22). Salamanca: Ediciones Amaru.

Nieto Bedoya, M. (2006). La educación de párvulos en la segunda mitad del siglo XIX: Palencia, un ejemplo singular. Revista de Educación, (340), 809-846.

Rodríguez Salcedo, S. (1949). Historia de los Centros Palentinos de Cultura. Publicaciones de la Institución Tello Téllez de Meneses, (2), 13-112.

Tiana Ferrer, A., Ossenbach Sauter, G. y Sanz Fernández, F. (coord.) (2002). Historia de la Educación. Edad Contemporánea. Madrid: UNED. 